\title{
Definitions of osteoarthritis in the knee and hand
}

\author{
Lennart TH Jacobsson
}

Osteoarthritis is the most common joint disorder, with a prevalence that increases with age. In 80 year old women radiologically defined osteoarthritis is found in over $70 \%$ in the distal interphalangeal joints (DIP) and in approximately $50 \%$ in the knees. ${ }^{1}$ Osteoarthritis is characterised by pain on movement, and the pathophysiological processes can be visualised radiologically. Radiographic findings include degenerative features (marginal osteophytes, subchondral cysts, loose bodies), reparative features (marginal osteophytes, subchondral sclerosis), and inflammatory features (joint effusions, bursar extension). Based on these features several radiological criteria have been developed for both the knees ${ }^{23}$ and hands. ${ }^{2-5}$ Recently the American Colleges of Rheumatology also have suggested clinical criteria for osteoarthritis of the knee, ${ }^{6}$ hip, ${ }^{7}$ and hand. ${ }^{8}$ The clinical criteria were chiefly developed to enable correct classification of patients in clinical studies, whereas radiological criteria can be used both for diagnosis and evaluation of disease progress. In most published reports, a rating of grade 2 or more (definite osteophytes and possible joint space narrowing) on the 0-4 graded radiographic scale developed by Kellgren and Lawrence ${ }^{2}$ has been used as the gold standard for diagnosis. Despite a positive correlation between the Kellgren-Lawrence scale and pain, only $55-85 \%$ and $11-28 \%$ of those with grade 3-4 changes in the knees and hands, respectively, have symptoms of pain.'

When reviewing and developing criteria for diagnosis and progression in osteoarthritis several characteristics should be considered. The criteria should be associated with symptoms, reflect the disease process, and predict long term outcome. For scientific studies they should also be accurate, reproducible, convenient to use, and relatively inexpensive.

\section{Knee osteoarthritis}

RADIOGRAPHIC CRITERIA

Knee radiographs for the diagnosis of osteoarthritis should be antero-posterior, bilateral, weight bearing, and taken with the patient in a standing position with the toes pointing straight ahead and with equal weight on both feet.

The first standardised way to determine knee osteoarthritis radiographically was developed by Kellgren and Lawrence in $1957,{ }^{2}$ and later became the accepted grading scale by the World Health Organisation in $1961 .^{10}$ The grading scheme defines four different levels of osteoarthritis:

Department of Rheumatology, Malmö University H pspital, S-205 02 Malmö, Sweden

L T H Jacobsson
- Grade 1: Doubtful narrowing of joint space and possible osteophytic lipping,

- Grade 2: Definite osteophytes and possible narrowing of joint space,
- Grade 3: Moderate multiple osteophytes, definite narrowing of joint space, and some sclerosis and possible deformity of bone ends, - Grade 4: Large osteophytes, marked narrowing of joint space, severe sclerosis, and definite deformity of bone ends.

This grading scheme has been widely used in population studies and therefore is still important when comparing prevalence rates, especially if one wishes to do comparisons with historical studies. Furthermore the scheme is fairly simple, not time consuming, and has been shown to be reproducible with intrarater and interrater correlations of over 0.8 in several studies. ${ }^{211}$ The major drawback is that osteophytes are considered a pathognomic feature for osteoarthritis, and this has been challenged by longitudinal studies. ${ }^{412}$ Furthermore it is obvious that it is narrowing in combination with symptoms, and not osteophytes, that eventually leads to operative intervention. It is therefore not surprising that only $33 \%$ of individuals with knee osteoarthritis deteriorated over an 11 year period according to the Kellgren-Lawrence scale, whereas $50 \%$ did so using a more sensitive scale. ${ }^{13}$

In 1968 Ahlbäck from Sweden introduced a radiographic definition that was based chiefly on joint space narrowing. ${ }^{14}$ This standard was developed from investigations of a series of 1800 knee radiographs and has frequently been used in northern Europe. The Ahlbäck scale only takes into account joint space narrowing and attrition, which is differentiated on a four graded scale. Although the scale, in similarity with the Kellgren Lawrence scale, is crude it has been shown to detect progression longitudinally $\left({ }^{16}\right.$, and Sahlström personal communication). A drawback may be that it does not take into account other features of osteoarthritis like osteophytes, sclerosis, cysts, and deformity.

Altman $e t a l^{4}$ evaluated a method of scoring knee osteoarthritis according to the following individual radiological features: joint space narrowing (0-3), osteophytes (0-3), sclerosis $(0-3)$, alignment $(0-3)$, and bony attrition $(0-3)$. Thirty two pairs of knees were evaluated both cross sectionally and longitudinally. Both the intrarater $(r=0.80)$ and interrater $(r=$ 0.76 ) variability for progression of medial knee osteoarthritis were best when calculating a combined score of all five features. Almost as good correlations were achieved when computing a combined score of narrowing, osteophytes, and sclerosis, which for simplicity was the recommended method by the authors. Of the individual features of medial knee osteoarthritis, narrowing and osteophytes had better interrater reliability than sclerosis, alignment, or bony attrition, both cross sectionally ( 0.68 to $0.74 v 0.40$ to 0.57$)$ and for detecting progression ( 0.54 to $0.60 v 0.34$ to 
0.52). This study represents a first attempt to compare the methodological properties of the various features of osteoarthritis. The comparison favours a combined score of several features, but in contrast to the Kellgren-Lawrence score it gives them equal weight. Recently a radiographic atlas with a similar variability in scoring but containing even more individual radiographic features has been published by Altman and coworkers. ${ }^{17}$

Spector $e t a l^{\beta}$ developed a scoring system for individual features of knee osteoarthritis based on features in both the tibiofemoral and the patellofemoral joints. These included tibiofemoral joint osteophytes (0-3), joint space narrowing (0-3), sclerosis (0-1), cortical collapse $(0-1)$, patellofemoral joint space narrowing (0-3), and osteophytes (0-3). When originally evaluated this standard revealed slightly lower intrarater and interrater agreement than with the studies above ${ }^{18}$ However, it includes evaluation of the patellofemoral joint, a joint whose importance has probably has been underestimated previously. In a recent population study, osteoarthritis in this joint was found to be more common and more strongly associated with disability then medial tibiofemoral osteoarthritis. ${ }^{19}$ Using this yardstick it has also been possible to demonstrate cross sectionally that osteophytes in knee osteoarthritis have a stronger association with joint pain then joint space narrowing. According to others, joint space narrowing may on the other hand may be a better predictor of progression than osteophytes for knee osteoarthritis. ${ }^{4}$

CLINICAL CRITERIA

In 1986 the American Rheumatism Association (ARA) developed clinical criteria for knee osteoarthritis. ${ }^{6}$ In the process numerous historical features, physical signs, laboratory tests, and radiographic features were evaluated in patients with clinical knee osteoarthritis and in a control group of which half had rheumatoid arthritis (RA). The combination of findings with the highest sensitivity and specificity was one consisting of knee pain and radiographic osteophytes and one of the following: more than 50 years old, morning stiffness of less than 30 minutes duration, and crepitus on active motion of the knee. ${ }^{6}$ These criteria have been criticised for circularity and because the control group was younger and to a large extent consisted of patients with rheumatoid arthritis, which might account for the fact that osteophytes and high age, and not joint space narrowing, were defined as good discriminative features. ${ }^{20}$ Hence, these criteria need further validation before being widely used in scientific studies.

\section{Hand osteoarthritis}

RADIOGRAPHIC CRITERIA

A single postero-anterior radiograph of both hands and wrists is adequate for scoring radiographic hand osteoarthritis.

In addition to developing criteria for knee osteoarthritis, Kellgren and Lawrence also developed criteria for osteoarthritis in other diarthrodial joints, including those of the digits and the wrist. $^{2}$ As with the scheme for knee osteoarthritis, abnormal findings were graded on a 1-4 scale, requiring the presence of definite osteophytes but not of definite joint space narrowing for a joint to be classified as grade 2 . The authors ${ }^{2}$ reported a high intrarater $(r=0.81)$ and interrater $(r=0.73)$ reliability, results that have later been confirmed as the criteria became widely used in population studies. ${ }^{51}$ As in the case of knee osteoarthritis, this standard considers osteophytes as a pathognomic feature of osteoarthritis, whereas it is clinical experience that other features of the disease, such as joint space narrowing, sclerosis, and deformity, may exist in the absence of osteophytes.

Altman et $a l^{t}$ instead applied individual radiographic features to evaluate radiographic hand osteoarthritis. Features evaluated were narrowing, osteophytes, erosions, and alignment, which were all found to have a reasonably high interrater $(r>0.74$ for the digits, $r>0.60$ for the wrist, cross sectionally) and intrarater reliability $(r>0.61$ for the digits, $r>$ 0.47 for the wrist, for progression). A combined score of narrowing, osteophytes, and erosions had the best overall diagnostic characteristics. Furthermore, it was shown that similar results were obtained when evaluating only the second and third distal (DIP) and proximal (PIP) interphalangeal joints, together with the trapezometacarpal joints bilatererally, as when evaluating all joints of the hand and wrist.

Kallman and coworkers developed similar grading scales for individual features of hand osteoarthritis ${ }^{5}$ using the five DIP joints, four PIP joints, the first metacarpalphalengeal joint, and the trapezoscaphoid joint of both hands. They found an intrarater and interrater reliability of the same magnitude as Altman et $a l,{ }^{4}$ both cross sectionally and longitudinally for narrowing (0-3), osteophytes (0-3), sclerosis (0-1), lateral deformity $(0-1)$, and cortical collapse $(0-1)$. Subchondral cysts $(0-1)$ were found to be a less reliable sign. The same investigators also showed in a longitudinal analyses of repeated hand radiographs on 177 men that joint space narrowing and doubtful osteophytes are the earliest signs of hand osteoarthritis regardless of age. ${ }^{22}$ This set of individual radiographic features has recently been validated and converted to a global scale $(0-2)$ of hand osteoarthritis by Lane and coworkers. ${ }^{23}$ According to this global scheme:

- grade 0 is normal,

- grade 1 represents mild osteophytes and/or mild narrowing and,

- grade 2 moderate-severe osteophytes and/or moderate-severe narrowing

Recently, representatives from these groups have published a radiographic atlas with a similar 0-3 grading of osteophytes, narrowing, slerosis, and malalignment of the DIP, PIP, and trapeziometacarpal joints. ${ }^{17}$

\section{CLINICAL CRITERIA}

The American College of Rheumatology has also developed clinical criteria for hand osteoarthritis, using similar methodology to 
that developed for knee osteoarthritis. ${ }^{8}$ Using the traditional format, the combination of symptoms, signs, and radiographic findings that had the highest sensitivity and specificity was pain, aching, or stiffness in the hand in combination with three of the following features: hard tissue enlargement of two or more of 10 selected joints, hard tissue enlargement of two or more DIP joints, fewer than three swollen MCP joints, and deformity of at least one of 10 selected joints.

\section{Conclusion}

We are still lacking a generally accepted definition of osteoarthritis of the knee and hand, and the grading scale developed by Kellgren and Lawrence 40 years ago remains in use. During the last decade major progress have been made in developing radiographic grading scales for diagnosis and staging of osteoarthritis that give equal weight to the individual features of the disease and do not consider osteophytes as pathognomic. Further development and validation of these scoring systems is needed before they will be universally accepted, including investigation of their association with symptoms and their ability to predict disease progress and the need for operative intervention. It also seems likely that different weight will be given to individual features of osteoarthritis, for example narrowing and osteophytes, when developing global scores for the the disease in the knee and the hand.

Standard radiographs have remained the most important technique for evaluation, since they are relatively convenient, inexpensive, and reflect major pathophysiological features of the disease. In addition, they appear to allow detection of disease progress, if sensitive scoring techniques are used. Despite this recent development, radiographic criteria for osteoarthritis still lack sensitivity in detecting early pathological changes and still to some degree lack sensitivity in detecting progress of the disease. Methods like ultrasound, scintigraphy, and magnetic resonance imaging may be better for detecting such changes. In the future it is therefore not unlikely that scoring systems based on these methods may be developed and used in scientific studies.
1 van Saase JLCM, van Romunde LKJ, Cats A, Vadenbroucke JP, Valkenburg HA. Epidemiology of osteoarthritis: Zoetermer survey. Comparison of radiological osteoarthritis in a Dutch population with that in 10 other
populations. Ann Rheum Dis 1989;48:271-80.

populations. Ann Rheum Dis 1989;48:271-80.

Kellgren JH, Lawrence JS. Radiologic assessment of osteoarthrosis. Ann Rheum Dis 1957;16:494-502.

3 Spector TD, Cooper C, Cushnaghan, Hart DJ, Dieppe PA. $A$ radiographic atlas of knee osteoarthritis. London: SpringerVerlag, 1992.

4 Altman RD, Fries JF, Bloch DA. Radiographic assessment of progression of osteoarthritis. Arthritis Rheum 1987; 31:1214-25.

5 Kallman DA, Wigley FM, Scott WW, Hochberg MC, Torbin JD. New radiographic grading scales for osteoarthritis of the hand. Arthritis Rheum 1989;32:1584-91.

6 Altman R, Fries JF, Bloch DA, Asch E, Block D, Bole G, et al. Development of criteria for the classification and reporting of osteoarthritis of the knee. Arthritis Rheum 1986;29:1039-49.

7 Altman R, Alarcon G, Appelrough D, Bloch D, Borenstein $\mathrm{D}$, Brandt $\mathrm{K}$, et al. The American College of Rheumatology criteria for the classification and reporting of osteoarogy criteria for the classification and reporting of oste
thritis of the hip. Arthritis Rheum 1991;34:505-14.

8 Altman R, Alarcon G, Appelrough D, Block D, Borenstein $\mathrm{D}$, Brandt $\mathrm{K}$, et al. The American College of Rheumatology criteria for the classification and reporting of osteoarthritis of the hand. Arthritis Rheum 1990;5:505-14.

9 Lawrence JS, Bremner JM, Bier F, Osteoarthritis. Prevalence in the population and relationship between symptoms and x-ray. Ann Rheum Dis 1966;25:1-24.

10 Kellgren JH. Atlas of standard radiographs. The epidemiology of chronic rheumatism. Oxford: Blackwell, 1963.

11 Felson DT, Naimark A, Anderson J, Kazis L, Castelli W, Meenan RF. The prevalence of knee osteoarthritis in the elderly: The Framingham osteoarthritis study. Arthritis Rheum 1987;30:914-8.

12 Hernborg J, Nilsson BE. The relationship between osteophytes in the knee joint, osteoarthritis and aging. Acto Orthop Scand 1973;44:69-74.

13 Spector TD, Dacre JE, Harris PA, Huskinsson EC. Radiological progression of osteoarthritis: an 11 year follow up study of the knee. Ann Rheum Dis 1992; follow up $1107-10$.

14 Ahlbäck S. Osteoarthrosis of the knee. Acta Radiol 1968:suppl 277.

15 Bauer G. Gonarthosis: classification, natural history and therapy. $f$ Rheumatol 1983;10(suppl 9):81-2.

16 Hernborg JS, Nilsson BE. The natural course of untreated osteoarthritis of the knee. Clin Orthop 1977;123:130-7.

17 Altman RD, Hochberg M, Murphy WA, Wolfe F, Lequesne M. Atlas of individual radiographic features of osteoarthri-
tis. Osteoarthritis and Cartilage 1995;3(suppl A):3-70.

18 Spector TD, Hart DJ, Byrne J, Harris PA, Dacre JE, Doyle DV. Definition of osteoarthritis of the knee for epidemiological studies. Ann Rheum Dis 1993;52:790-4.

19 McAlindon TE, Snow S, Cooper C, Dieppe PA. Radiographic patterns of osteoarthritis of the knee joint in the community; the importance of the patellofemoral joint. Ann Rheum Dis 1992;51:844-9.

20 McAlindon T, Dieppe PA. Osteoarthritis: definitions and criteria. Ann Rheum Dis 1989;48:531-2.

21 Hochberg MC, Lethbridge-Cejku M, Scott WM, Plato CC, Tobin JD. Appendicular bone mass and osteoarthritis of the hands of women: data from the Baltimore longitudinal study of aging. $\mathcal{F}$ Rheumatol 1994;21:1532-6.

22 Kallman DA, Wigley FM, Scott WW, Hochberg MC, Tobin JD. The longitudinal course of hand osteoarthritis in a male population. Arthritis Rheum 1990;33:1323-32.

23 Lane NE, Nevitt MC, Genant HK, Hochberg M. Reliability of new indices of radiographic osteoarthritis of the hand, hip, and lumbar disc degeneration. I Rheumatol 1993; 20:1911-7. 